RU9226

\title{
Geometry, Scaling and Universality in the Mass Distributions in Heavy Ion Collisions
}

\author{
A. Z. Mekjian \\ Deparment of Physics \& Astronomy \\ Rutgers University, Piscataway, New Jersey 08855-0849 \\ S. J. Lee \\ Department of Physics, Kyung Hee University, Suwon 449-701, Korea. \\ (Received Physical Review C)
}

\begin{abstract}
Various features of the mass yields in heavy ion collisions are studied. The mass yields are discussed in terms of iterative one dimensional discrete maps. These maps are shown to produce orbits for a monomer or for a nucleus which generate the mass yields and the distribution of cluster sizes. Simple Malthusian dynamics and nonlinear Verhulst dynamics are used to illustrate the approach. Nuclear cobwebbing, attractors of the dynamics, and Lyapanov exponents are discussed for the mass distribution. The self-similar property of the Malthusian orbit offers a new variable for the study of scale invariance using power moments of the mass distribution. Correlation lengths, exponents and dimensions associated with scaling relations are developed. Fourier transforms of the mass distribution are used to obtain power spectra which are investigated for a $1 / f^{\beta}$ behavior.
\end{abstract}

PACs Numbers: 24.60.-k, 25.70.Np, 47.20.Ky, 02.90.+p

Typeset Using REVTEX 


\section{INTRODUCTION}

The purpose of this paper is to illustrate how simple pictures developed from soluble models [1,2, 3, 3] can lead to useful methods in understanding the mass yields in heavy ion collisions. First, we will attempt to show how iterative one dimensional maps [ [由,5,6] can characterize ensemble averaged behaviors of these yields. The language of one dimensional maps contains such ideas as cobwebbing, orbits and trajectories, attractors, basins of attraction, Lyapanov exponents, non-linear dynamics and chaos [2, 3, 4, 5, 6, 6, 8, 9]. The approach taken here will also suggest new ways of portraying the experimental data which go beyond any specific model discussed here and which may give further insights into the dynamics of the collision. One main result of this paper is that the mass yield can be generated by the orbit of a monomer or of a nucleus (as the initial point) in its approach to an attractor. Simple Malthusian dynamics with its associated geometric growth will be shown to characterize some features of the data considered in this paper. This geometric Malthusian model will be shown to be an approximate representation of the soluble models considered in Refs. [1-3]. A non-linear Verhulst map will also be considered to investigate signals for non-linear dynamics and limitations to unbounded geometric growth.

In Refs. [1-3] we have developed an exactly soluble model of fragmentation and obtained an evolution equation [2] which can be reduced to a Fokker-Plank equation for a mean cluster distribution. The evolution equation relates the change of the mean cluster number of a given size to the mean cluster number of all the other sizes when the parameter $x$ (introduced in Ref. [1] and discussed below) or equivalently the temperature changes [2]. The solution of this evolution equation at a given $x$ value determines the mean distribution at the corresponding temperature. We have also shown [2,3] that our $x$ model has a quite similar structure to a Markov population process [7]. These properties of our fragmentation model enable us to relate this model with one dimensional maps for the mean behavior in a fragmentation process. The discrete cluster size in a nuclear fragmentation corresponds to the iterative index of a discrete map. 
Then, secondly, we will discuss the scaling and self-similar behavior of the power moments [8] that can be obtained from the mass yield produced in a nuclear collision. Since a simple linear geometric map generates orbits having self-similar properties, power moments of the orbit points also will have a scaling power law behavior. The self similar property of the geometric or Malthusian model will be shown to offer a new variable for the study of scale invariance and universality in the power moments of the mass yield. Moreover, some analytic results for the scaling properties of the soluble model considered in Refs. [1,2],3] will be given and compared to the simpler geometric model. The scaling properties of the power moments with bin size have associated correlation lengths and exponents related to the dimension of the system as discussed in Ref. [8] and further developed here.

Thirdly, this paper will investigate the Fourier spectrum of the fragmentation distribution. The importance of the Fourier spectrum is the appearance of a power law behavior with frequency $f$, such as $1 / f^{\beta}$ behavior, with $\beta$ an exponent which, in a description of noise spectra, characterizes the type of noise [9]. For example $\beta=0$ is white noise, and $\beta=1,1 / f$ noise. Finally, we will investigate some experimental data of Ref. [10].

\section{GEOMETRICAL ITERATIVE MAPS AND MEAN CLUSTER DISTRIBUTIONS}

The interrelationships between one dimensional maps and mass yields developed out of a previous model of fragmentation and its generalizations [1,2, [2]. Specifically, the model of Refs. [1,2,3] contains a simple expression for the ensemble averaged mean distribution of cluster sizes $Y_{A}(k, x)=<n_{k}>$ given by

$$
<n_{k}>=\frac{x}{k} \frac{A !}{(A-k) !} \frac{\Gamma(x+A-k)}{\Gamma(x+A)},
$$

where the $\Gamma$ 's are gamma functions. The $A$ is the total number of nucleons, $k$ the size of the cluster, and the $x$ is a tuning parameter which can vary from 0 to infinity depending on the temperature. The parameter $x$ contains the dynamical quantities associated with the fragmentation. Thermodynamic arguments [1,22,3] give 


$$
x=\frac{V}{v_{o}} \exp \left[-\left(\frac{a_{v}}{k_{B} T}\right)-\left(\frac{k_{B} T}{\epsilon_{o}}\right)\left(\frac{T_{o}}{T+T_{o}}\right)\right] .
$$

The $V$ is the freeze out volume, $T$ is the equilibriation temperature, and $v_{o}$ is the quantum volume $v_{o}=h^{3} /\left(2 \pi m_{p} k_{B} T\right)^{3 / 2}$ with $m_{p}$ the mass of a nucleon. The $a_{v}$ is the coefficient in a simplified equation for the binding energy $E_{B}$ of a cluster of $k$ nucleons: $E_{B}(k)=a_{v}(k-1)$. The $\epsilon_{o}$ is the level density parameter related to the spacing of excited levels in a cluster and $T_{o}$ is a cut-off temperature for internal excitations. Further details for this relation can be found in Refs. [1, 2, 3]. Here, we mention the following. Small $x$ corresponds to a low temperature and/or small interaction volume and large $x$ corresponds to a high temperature and/or large interaction volume. Also for small $x$, the $\left\langle n_{k}>\sim x\right.$ and thus the $\left\langle n_{k}>\right.$ depend on the separation energy $a_{v}$ in the Boltzmann factor $\exp \left(-a_{v} / k_{B} T\right)$, For large $x, x>>A$, the $<n_{k}>\sim A(A / x)^{k-1}$ and thus $<n_{k}>\sim \exp \left[a_{v}(k-1) / k_{B} T\right]=\exp \left[E_{B}(k) / k_{B} T\right]$. In this limit $\left\langle n_{k}>\right.$ depends on the Boltzmann factor in total binding energy. The $<n_{k}>$ given by Eq.(1) thus contains both the evaporation region dominated by separation energies and the multifragmentation region dominated by binding energy factors [3]. As a final remark on the physical significance of $x$, we note that the exponent $-k_{B} T T_{o} / \epsilon_{o}\left(T+T_{o}\right)$, which also appears as a Boltzmann factor, is the temperature dependent part of the Helmholtz free energy $F$ per particle at low temperatures divided by $k_{B} T$ [2]. In particular, $F=$ $\left(3 N \epsilon_{F} / 5\right)\left(1-5 \pi^{2}\left(k_{B} T / \epsilon_{F}\right)^{2} / 12\right)$ where $\epsilon_{F}$ is the Fermi energy which is related to the level spacing $\epsilon_{o}$ via $\epsilon_{o}=4 \epsilon_{F} / \pi^{2}$. Note, for low $T, T_{o} /\left(T+T_{o}\right)=1$ and the dominant term in the factor $\exp \left[\left(-a_{v} / k_{B} T\right)-k T / \epsilon_{o}\right]$ is the separation energy part, ie., $\left(-a_{v} / k_{B} T\right)$. For large $T$, the factor $\exp \left[-k_{B} T T_{o} / \epsilon_{o}\left(T+T_{o}\right)\right]$ in $x$ gives rise to the exponential behavior of the internal partition function of a cluster evaluated in a Fermi gas approximation. Thus, the factor $\exp \left[-k T T_{o} / \epsilon_{o}\left(T+T_{o}\right)\right]$ evolves from an evaporation factor into a Boltzmann enhancement factor for internal excitations. This behavior is similar to the evolution of the separation energy into a factor for the binding energy of a cluster from the $\exp \left(-a_{v} / k_{B} T\right)$ part of $x$. In the rest of this paper, we will keep $x$ as an unspecified tuning parameter. The above remarks show that this tuning parameter contains the underlying physical quantities: $V, T$, 
$\epsilon_{o}$ and $a_{v}$.

The simple $x$-model of Eq.(11) fits the smooth behavior of the fragment distribution in various proton-nucleus and nucleus-nucleus collisions [11]. However this model does not describe all the detailed behavior of these distributions such as fission peaks and shoulders [11,12]. One way of overcoming this limitation is using a size $k$ dependent parameter $x_{k}$ instead of a single parameter $x$. The size dependence of $x_{k}$ may arise from a more realistic treatment of the binding energy by including surface and Coulomb energies, and shell effects which were not included in obtaining Eq.(11). However, when this is done, we loose the exact solubability of the model. Another way of overcoming this limitation is to seek an alternate methods of approach for fragmentation process. Here, we will show that the result of Eq.(11) has an interpretation in terms of iterative one dimensional maps and use this interrelation as a starting point for another type of description of the complex dynamics of the fragmentation process. Iterative maps have been used to model the behavior of complex systems in many other areas [6].

The $x$ model of Eq.(11) has a recursive or iterative property. Namely, the total average masses in clusters of size $k$, which is given by $m_{k}=k<n_{k}>$, can be related to the total masses of clusters of size $k+1$ by

$$
m_{k+1}=r_{A}(k, x) m_{k} .
$$

Here we dropped the broken bracket representing an ensemble average of $m_{k}$ for simplicity in notation. The recursive coefficient $r_{A}(k, x)$ is simply

$$
r_{A}(k, x)=\frac{(A-k)}{(A-k+x-1)} \equiv e^{\lambda_{A}(k, x)} .
$$

The initial point in the $x$-model is $m_{1}=x A /(A+x-1)$. The mass distribution then follows by successive iterations,

$$
m_{1} \rightarrow m_{2} \rightarrow m_{3} \rightarrow \cdots \rightarrow m_{k} \rightarrow \cdots \rightarrow m_{A}
$$

and satisfies the constraint $A=\sum_{k} m_{k}$. 
We can also consider the backward version of the forward invertible mapping given by $\mathrm{Eq} \cdot(3)$;

$$
\begin{aligned}
m_{A-k} & =\tilde{r}_{A}(k, x) m_{A-k+1}, \\
\tilde{r}_{A}(k, x) & =1 / r_{A}(A-k, x) .
\end{aligned}
$$

Since Eq.(3) is a linear invertible map, this backward map is the inverse map of the forward map and gives the sequence of

$$
m_{A} \rightarrow m_{A-1} \rightarrow \cdots \rightarrow m_{k} \rightarrow \cdots \rightarrow m_{1}
$$

The sequence of Eq.(5) gives the orbit of $m_{1}$ under iteration and the recursive relationship generates pairs $\left(m_{k}, m_{k+1}\right)$ constructed by the recursive coefficient. This way of representing the results of the above model is similar to that used in studies of population dynamics and, in particular, to models in which the population evolves in discrete steps. The cluster size $k$ serves as the discrete index and corresponds to the successive generations while the mass distribution $m_{k}$ is the analogue of the population size in the $k$-th generation. The $k$ runs from 1 to $A-1$ and the orbit should be eventually normalized so that $\sum_{k=1}^{A} m_{k}=A$.

In a previous paper [13] another connection of the $x$-model of fragmentation with a model in population genetics due to Ewens [14] was explored. There the index $k$ represented the number of times of a particular gene appears and $n_{k}$ the number of different types of genes each appearing $k$ times in a sample of size $A$. The parameter $x$ is connected with the mutation rate and effective population size in population genetics and evolutionary biology.

Using the analogy with population dynamics, the data on the mass distribution can be plotted as points for each pair $\left(m_{k}, m_{k+1}\right)$ for each $k=1, \ldots, A-1$ with $m_{k+1}$ along the vertical axis and $m_{k}$ along the horizontal axis. A smooth curve through the data gives the recursion curve for that data set. For, example, the simple Malthus model in population dynamics, which is

$$
m_{k+1}=r m_{k}=r^{k} m_{1}=r^{k+1} m_{0},
$$


gives a straight line for $r \neq 1$ in such a plot. Here $m_{0}=m_{1} / r$ and the constraint $\sum_{k=1}^{A} m_{k}=$ $A$ determines $m_{1}=A(1-r) /\left(1-r^{A}\right)$. The parameter $r$ can be determined by the data set by a least square fit and $r$ represents the slope of a straight line fit. The parameter $r$ can also be determined by the slope $\lambda=\log r$ of a straight line fit in a $\log m_{k} v s k$ plot. In general this curve will be more complex than a straight line. It can depend on $k$ as in Eq.(四) for the $x$-model or can depend on $m_{k}$ itself as in a non-linear map. The orbit of $m_{1}$ upon successive iteration is determined by $m_{k+1}=r^{k} m_{1}$ in the Malthus model.

It should be noted that when the $k$ dependence of $r_{A}(k, x)$ is averaged over then the fragmentation model of Eq.(3) reduces to the simple Malthus model. For example, a geometric mean $\bar{r}$ can be defined by

$$
\begin{aligned}
\bar{r}=e^{\bar{\lambda}} & =\left[r_{A}(1, x) r_{A}(2, x) \cdots r_{A}(A-1, x)\right]^{1 /(A-1)} \\
& =\exp \left(\left[\lambda_{A}(1, x)+\lambda_{A}(2, x)+\cdots+\lambda_{A}(A-1, x)\right] /(A-1)\right) .
\end{aligned}
$$

The $\bar{\lambda}$ is the arithmetic mean of the $\lambda_{A}(k, x)=\ln r_{A}(k, x)$. In the Malthus model or in the fragmentation model of Eq.(3i) when $r>1$ or $x<1, m_{k}$ increases geometrically with increasing $k$ and when $r<1$ or $x>1, m_{k}$ decreases geometrically with increasing $k$. As we shall see, the mass distribution of cluster sizes in the back direction, i.e., $k$ near $A$, is an important quantity.

Iterative models also lend themselves to a geometrical iteration known as cobwebbing which involves the generation of the pairs $\left(m_{k}, m_{k+1}\right)$ by graphical means. Starting with $m_{1}$ on the $x$-axis, $m_{2}$ is evaluated as the corresponding $y$-axis value on the curve, $y=r x$, or $m_{2}=r m_{1}$. Then $m_{2}$ is reflected onto the $x$-axis through the $45^{\circ}$ line, $y=x$. Next $m_{3}$ is generated by repeating the procedure with $m_{2}$ on the $x$-axis and so on. The cobwebbing gives a useful visual way of understanding the trajectory or orbit and gives a large amount of information by pure geometrical means and simple graphical analysis.

The behavior of $m_{k}$ as $k$ becomes large ( $k$ is limited by $A$ in a nuclear fragmentation) gives the attractors of the orbits. In Malthusian dynamics, all orbits of $m$ will go asymptotically to the $+\infty$ attractor or to the 0 attractor if $r>1$ or $r<1$ respectively. The point $m=0$ is 
an attracting fixed point for $r<1$ and a repelling fixed point for $r>1$. At $r=1$ and from the result of Eq.(4) with $x=1$, the orbit of $m$ stays fixed for all values of $m$. For this case, $m_{k+1}=m_{k}$ forms a fixed line of the map.

Figs.1(a) - 1(d) illustrate an application of the above ideas to the experimental data in nuclear fragmentation. From the fits shown, we see that the fragmentation model of Eq.(1) with $x=0.3$ (solid line) and its simple Malthusian version (dashed line) give a reasonably good fit to the data.

These data show that the number of clusters decreases as the size $k$ increases for small $k$, then slowly increases until it reaches a somewhat flat region (also called a "shoulder") at large $\mathrm{k}$, then finally rises sharply again to $k=A$. The linear map of Eq.(8) fits the data better than the $x$-model of Eqs.(四) and (3) except for the $p+A u$ data of Fig.1(c). Allowing a simple $k$ dependence in the coefficient $r$ as $r(k)=a+b k$ with $a=0.9833$ and $b=0.000333$, the linear Malthus map can fit the whole region of the $p+A u$ data (dash-dotted line in Fig.1(c)), (using a $k$ dependent $r$ is similar to mixing various $r$ values as we will consider in Section VI for the $\mathrm{Au}$ emulsion data [10]). This simple $k$ dependent linear map has a geometric mean $\bar{r}$ of 1.016 and a minimum $n_{k}$ at $k \approx 85$. However both the $x$-model and the linear Malthus map do not produce a levelling off at a large mass region which may be related to a non-linear map as we will discuss in the next section.

\section{NONLINEAR MAP}

Non-linear models were introduced to limit the geometric growth of the Malthus model (attractor at $\infty)$. The classic examples are the Verhulst models; two examples are

$$
m_{k+1}=\frac{R_{V} m_{k}}{m_{k}+K_{V}}
$$

and the logistic map,

$$
m_{k+1}=R_{V} m_{k}\left(K_{V}-m_{k}\right)
$$


with the $K_{V}-m_{k}=0$ if $m_{k}>K_{V}$. The result of Eq.(11) can be rewritten as $P_{k+1}=$ $a P_{k}\left(1-P_{k}\right)$. The $K_{V}$ is the carrying capacity of the system. The logistic map Eq.(11) has two fixed points, $m=0$ and $m=K_{V}-1 / R_{V}$. Two fixed points of the Verhulst map of Eq.(10) are $m=0$ and $m=m_{s}=R_{V}-K_{V}$. These fixed points of Eqs.(10) and (11) are all finite in contrast to the Malthus map Eq.(8). Since nuclei around $k=230$ spontaneously fission, we expect a limit on the iteration number $k, k_{\max } \approx 230$, when we consider nuclear fragmentation. It should also be noted that in the $x$-model the iteration is truncated after $k=A-1$ since $r_{A}(k=A, x)=0$.

The Verhulst model given by Eq.(10) can be solved analytically because the inverse mass $L_{k}=1 / m_{k}$ obeys a linear mapping

$$
L_{k+1}=\frac{1}{R_{V}}+\frac{K_{V}}{R_{V}} L_{k} .
$$

Due to this linearity, the Verhulst map has a unique inverse map and thus both the forward map and the backward map generate the same orbit once the end points $(k=1$ and $k=A)$ are the same.

The resulting distribution of cluster sizes arising from the orbit of $m_{1}=n_{1}$ is given by

$$
n_{k+1}=\left(\frac{1}{k+1}\right) \frac{m_{s}}{\left(1-a^{k}\right) n_{1}+a^{k} m_{s}} n_{1}
$$

where $a=K_{V} / R_{V}$ and $m_{s}=R_{V}-K_{V}$. The appearance of $a^{k}$ in Eq.(13) gives a geometric behavior to $n_{k+1}$. When $K_{V}>R_{V}, L_{k} \rightarrow \infty$ and $m_{k} \rightarrow 0$ geometrically. When $K_{V}<R_{V}$, $L_{k} \rightarrow 1 / m_{s}$ and $m_{k} \rightarrow m_{s}$, the saturating value (attractor) of the Verhulst mapping of Eq.(10). Note $n_{k}$ falls because of the extra $k$ in $n_{k}=m_{k} / k$. When $R_{V}=K_{V}$, then $a=1$ and $n_{k+1}$ no longer has a geometric part but rather falls as a power law:

$$
n_{k+1}=\left(\frac{1}{k+1}\right) \frac{1}{1+n_{1} k / R_{V}} n_{1} .
$$

For large $k$ such that $n_{1} k / R_{V}>>1$ the $n_{k+1} \sim R_{V} /(k+1) k$.

The $n_{k}$ has the interesting property of having a bifurcation point when $a=K_{V} / R_{V}=$ $e^{-\zeta}<1$. This behavior is not present in $m_{k}$ which increases uniformly with $k$ for $a<1$. 
The orbit of $m_{k}$ monotonically approaches one of the two fixed points ( 0 or $\left.m_{s}\right)$ depending on the value of $a$. The orbit of $n_{k}$ can have both minimum and maximum points depending on the value of $a$ and $m_{s} / m_{1}$. The bifurcation point arises from the extra $k$ in going from $m_{k} \rightarrow n_{k}=m_{k} / k$. Specifically when $m_{s} / m_{1}>e^{2-\zeta}+1$, the $n_{k}$ has a minimum at $k_{\min }$ and a maximum at $k_{\max }$ given by the solution to the transcendental equation $y_{1}=y_{2}$ with $y_{1}=\zeta(k+1)-1$ and $y_{2}=e^{\zeta k} /\left(m_{s} / m_{1}-1\right)$. The value $k_{\min }$ at which $n_{k}$ is a minimum is bounded from below by $1 / \zeta-1$ as $m_{s} / m_{1} \rightarrow \infty$. The $k_{1 / 2}$ point is defined as $m_{k_{1 / 2}+1}=m_{s} / 2$ and is given by $k_{1 / 2}=\left[\ln \left(m_{s} / m_{1}-1\right)\right] / \zeta$. At this point $k_{1 / 2}$, which is also the inflection point of $m_{k}$, the slope $d y_{1} / d k$ is equal to the slope $d y_{2} / d k$. Thus $k_{\text {min }} \leq k_{1 / 2} \leq k_{\max }$ with $k_{\min }=k_{1 / 2}=k_{\max }$ when

$$
m_{s} / m_{1}=e^{2-\zeta}+1
$$

Thus the condition Eq.(15) determines the bifurcation surface, i.e., the two roots $k_{\min }$ and $k_{\max }$ merge to $k_{1 / 2}=2 / \zeta-1$, in the parameter space of $\left(\zeta, m_{s}\right.$, and $\left.m_{1}\right)$.

Fig.1(d) also illustrates a fit using the non-linear mapping of Eq.(10) to the $p+A g$ data where the maximum point $k_{\max }$ is placed near the endpoint $k=A$ to fit the shoulder (dash-dotted curve). Comparing the result of the $x$-model of Eq.(1), the Malthus model,

and the Verhulst map near the endpoint with $k \approx A$, we see some differences. The result of Eq.(11) for $x<1$ approaches the endpoint with an upward cusp, the Malthus model comes in as a straight line with positive slope for $r>1$, and the Verhulst model can approach the endpoint with zero slope, positive slope, or negative slope depending on the position of $k_{\max }$ with respect to the endpoint. The data show a flat region or "shoulder" near the endpoint and then finally a cusp rise.

\section{SELF-SIMILARITY, SCALING BEHAVIOR AND UNIVERSALITY IN THE POWER MOMENTS OF MASS DISTRIBUTION}

In this section, we will study the behavior of the simple Malthus model of Eq.(8) in greater detail. The trajectory behavior of a Malthus map can also be seen by looking at a 
hypothetical two dimensional spiral

$$
m(r, \theta)=m_{1} r^{\theta / 2 \pi} e^{i \theta}=x+i y
$$

where $\theta$ can vary from 0 to $\infty$. The intersection of the continuous spiral with the positive real axis, i.e., $m(r, \theta)=x_{k}$ at the values of $\theta=2 \pi(k-1)$, gives the discrete set of points $x_{1}=m_{1}$ at $\theta=0, x_{2}=m_{2}=r m_{1}$ at $\theta=2 \pi$, and so on. The index $k$, representing the $k$-th intersection, is a labeling of clusters by size. The resulting points become the discrete "time" $(t=k)$ version of a continuous system $(t=\theta / 2 \pi)$ in two dimensions. For $r>1$ or $x<1$, the system spirals outward to a fixed point at infinity and for $r<1$ or $x>1$, the system spirals inward to a fixed point at zero. For $r=1$, the spiral reduces to a circle with a radius $m_{1}$. In a fragmentation process, $x=1$ corresponds to the point where the mass $A$ is uniformly distributed over the cluster index $k$. The quantities $\lambda_{A}(k, x)$ which appear in Eq.(4) can be considered as the Lyapanov exponents for each loop $k$ and $\bar{\lambda}=\ln \bar{r}$ the arithmetic mean Lyapanov exponent of the truncated spiral.

We note that the geometric growth in $m_{k+1}$ of Eq.(8) or $m(r, \theta)$ of Eq.(16) can also be interpreted in terms of a logarithmic spiral. Specifically, we consider the logarithmic spiral function $m(\theta)$ defined by the equation

$$
m(\theta)=m_{1} e^{\alpha \theta}=e^{\alpha\left(\theta+\log m_{1} / \alpha\right)} .
$$

The polar coordinates of the spiral are $(m, \theta)$ and $m_{1}$ and $\alpha$ are constants. The mass spectrum $m_{1}, m_{2}, \ldots, m_{k}, \ldots$ corresponds to the radial intercept of the spiral with the $x$ axis. The successive intercepts give the size index $k$. Thus $m_{2}=m_{1} e^{2 \pi \alpha}$ and consequently the coefficient $r=e^{2 \pi \alpha}$. The geometric meaning of $\alpha$ in terms of the logarithmic spiral can be obtained by considering $\alpha=d(\log m) / d \theta=(d m / d \theta) / m$. The cotangent of the angle between any radius vector (the $x$-axis considered as an intercept line here) and the tangent to the spiral at the intercept point is then equal to $\alpha$, i.e., $\cot \beta=\alpha$; the $\beta$ is independent of the orientation of the intercept radius vector (the $x$-axis here) and the branch of the spiral. Rotation of the intercept axis (the $x$-axis) by angle of $\theta_{0}$ corresponds to changes in $m_{1}$ to 
$m_{1} e^{\alpha \theta_{0}}$ and not in $r, \alpha$, or $\beta$. Secondly, it should be noted that changes in $r=e^{2 \pi \alpha}$ correspond to changes in $\alpha$ and thus in $\beta$ since $\alpha=\cot \beta$. As noted in Ref. [9], the logarithmic spiral is self-similar since $m_{1}$ can be absorbed into a constant angle $\log m_{1} / \alpha$ as shown in Eq.(17).

Self-similarity of the geometric sequence $1, r, r^{2}, r^{3}, \ldots$ generated by the coefficient $r$ is also discussed in Schroeder [9]. Figs.3.10 and 3.11 in Ref. 99 illustrates a triangular and a rectangular construction of this property of the infinite series $1+r+r^{2}+\cdots=1 /(1-r)$ for $r<1$. Extending the index $k$ to $-\infty \leq k \leq \infty$, the series has an exact self-similar structure. Thus the power moments of this $m_{k}$ as considered in Ref. [8] has a power law behavior in the bin size $L$. However if we restrict the set to be $k=1,2, \ldots, A$, then the power moment would have an $A$ dependence.

In a Malthus map the $q^{\prime}$ th order power moment of the mass distribution probability $p_{k}=m_{k} / A$ with bin size $L[8$ is given by

$$
\begin{aligned}
P_{q}(L, r) & =\sum_{J=1}^{[A / L]}\left[\sum_{k \in J} p_{k}\right]^{q}=\sum_{J=1}^{[A / L]}\left[\sum_{k \in J} r^{k} m_{1} / A\right]^{q} \\
& =\left[\frac{\left(1-r^{L}\right)}{\left(1-r^{A}\right)}\right]^{q}\left[\frac{1-r^{A q}}{1-r^{L q}}\right] .
\end{aligned}
$$

For $r \approx 1$, it is easy to show that $P_{q}(L, r)=(L / A)^{q-1}$. Fig.2 shows $P_{q}(L, r)$ with $r=$ $A /(A+x-1)$. Fig.3 shows the power moments of the mean mass distribution

$$
\bar{P}_{q}(L, x)=\sum_{J=1}^{[A / L]}\left[\sum_{k \in J}<p_{k}>\right]^{q}=\sum_{J=1}^{[A / L]}\left[\sum_{k \in J} \frac{k<n_{k}>}{A}\right]^{q}
$$

in $x$ model of Ref. [8] compared to $P_{q}(L, r)$ of Eq.(18). Here, the bar over $P_{q}$ signifies that the power of the mean is being taken. Below we will also evaluate the mean of the power. The slope in Fig.3 corresponds to the generalized dimension $D_{q}$ defined in Ref. [8],

$$
D_{q}(L)=\frac{1}{(q-1)} \frac{\log \left[\bar{P}_{q}(L, x)\right]}{\log (L / A)} .
$$

This figure shows that $D_{q}$ is about 1 for $x>1$ and much smaller than one with $q$ dependence for small $x$. We can rewrite Eq.(18) as

$$
P_{q}(L, r)=P_{q}(L, \lambda)=\left[\frac{\sinh (\lambda L / 2)}{\sinh (\lambda A / 2)}\right]^{q}\left[\frac{\sinh (q \lambda A / 2)}{\sinh (q \lambda L / 2)}\right],
$$


where $r=e^{\lambda}$. This rapidity type quantity $P_{q}(L, \lambda)$ of $\lambda L$, which is normalized to 1 at $L=A$, can be used as a new variable in a study of the power moments.

In the $x$ model, for the bin size $L=1$ and for $x<1$, using Stirling approximation for large $A>>x$,

$$
\Gamma(A+x) \approx(A+x-1)^{x-1} \Gamma(A+1)
$$

the power moment $\bar{P}_{q}(L, x)$ of the mean mass distribution becomes

$$
\begin{aligned}
\bar{P}_{q}(1, x) & =\sum_{k=1}^{A}\left[\frac{k<n_{k}>}{A}\right]^{q}=\sum_{k=1}^{A}\left[\frac{x}{A} \frac{A !}{(A-k) !} \frac{\Gamma(A+x-k)}{\Gamma(A+x)}\right]^{q} \\
& \approx\left[\frac{x}{A^{x}}\right]^{q}\left[(\Gamma(x))^{q}+\zeta((1-x) q)\right]
\end{aligned}
$$

where $\zeta(a)$ is a zeta function. For $(1-x) q=1$, the $\zeta((1-x) q)$ should be replaced by $\log (A-1)+0.57722$. Since $\bar{P}_{q}(A, x)=1$, then assuming the power moment follow a power law, we can write $\bar{P}_{q}(L, x)=(L / A)^{\alpha_{q}}$ with $\alpha_{q}=-\log \left[\bar{P}_{q}(1, x)\right] / \log (A)$ at low $x$. Then

$$
\alpha_{q}=q x-\frac{q \log [\Gamma(1+x)]+\log \left[1+(\Gamma(x))^{-q} \zeta((1-x) q)\right]}{\log A} .
$$

Usually, $\alpha_{q}$ is written as [8,9] $\alpha_{q}=D_{q}(q-1)$ so that the dimension $D_{q}$ is

$$
D_{q}=\frac{q x}{q-1}-\frac{q \log [\Gamma(1+x)]+\log \left[1+(\Gamma(x))^{-q} \zeta((1-x) q)\right]}{(q-1) \log A} .
$$

Fig.4 show the power moments $\bar{P}_{q}(L, x)$ for $x=0.5$. This figure also shows a fit with an $\alpha_{q}(L)=a_{q}+b_{q} / L$ form (thin solid lines) and a fit with the Stirling approximation of Eq.(23) for $q=2$ (thin dash-dotted line). The $D_{q}(L)$ of Eq.(20) are shown in Fig.4(b) for each $q$.

Moreover, for $x=m$ with $m$ an integer, the mean fractional occupancy in bin $J$ of length $L$ is given by $\sum k<n_{k}>/ A=\bar{P}_{J}(L, x=m)$, where the sum $\Sigma$ is over $k$ from $k=(J-1) L+1$ to $J L$. This mean fractional occupancy can be obtained from

$$
\bar{P}_{J}(L, x=m)=\frac{(A-1) ! m !}{(A+m-1) !} F_{J}(L, m)
$$

The $F_{J}(L, m)=H_{J-1}(L, m)-H_{J}(L, m)$ and, using Eq.(48) of Ref. [3]),

$$
H_{J}(L, m)=\frac{\left(m+A_{J}(L)-1\right) !}{\left(A_{J}(L)-1\right) ! m !} .
$$


The $A_{J}(L) \equiv A-J L$. At $x=m=1$, the $\bar{P}_{J}(L, 1)=L / A$ and at $x=m=2$, the $\bar{P}_{J}(L, 2)=(2 L / A)-(2 J-1)(L / A)^{2}$. The large $A$ Stirling limit of $\bar{P}_{J}(L, x)$ is

$$
\bar{P}_{J}(L, x) \cong\left(1-\frac{(J-1) L}{A}\right)^{x}-\left(1-\frac{J L}{A}\right)^{x} .
$$

With this limit, the power moment $\bar{P}_{q}(L, x=m)$ can be rewritten in terms of a simple convolution, namely

$$
\bar{P}_{q}(L, x=m)=\left(\frac{L}{A}\right)^{q} \sum_{J=1}^{n}\left(C_{J}(m)\right)^{q}
$$

with $n=A / L$ and $C_{J}(m)$ the discrete convolution

$$
C_{J}(m)=\sum_{k=0}^{m-1} b_{J-1}^{m-1-k} b_{J}^{k}
$$

Here $b_{J}=1-(J L / A)$. For $m=1, C_{J}(1)=1$ and for $m=2, C_{J}(2)=(n+1-2 J) / n$. The geometric features in $\bar{P}_{q}(L, x)$ arise when the factor $1-J L / A \simeq \exp (-J L / A)$. Specifically,

$$
\bar{P}_{J}(L, x)=\frac{\exp (-(J-1) L x / A)-\exp (-J L x / A)}{N(x)}
$$

with $N(x)=1-e^{-x}$ to guarantee that $\bar{P}_{J}$ is normalized to unity when summed over $J$. Then,

$$
\bar{P}_{q}(L, x) \equiv \sum_{J=1}^{n} \bar{P}_{J}^{q}(L, x)=\left[\frac{\sinh \left(\frac{L x}{2 A}\right)}{\sinh \left(\frac{x}{2}\right)}\right]^{q}\left[\frac{\sinh \left(q \frac{x}{2}\right)}{\sinh \left(q \frac{L x}{2 A}\right)}\right],
$$

which has the same structure of the geometric model of Eq.(21); $\lambda \leftrightarrow x / A$.

Since the Malthus model can describe only the mean behavior of the mass distribution, we can consider only the power of the mean distribution, Eq.(18). However for the $x$ model, as we have considered in Ref. [8], we can also study the ensemble average of power moments in each event. For a small "integer" $x$, we can evaluate the power moment,

$$
P_{q}(L, x)=\left\langle\sum_{J=1}^{[A / L]}\left[\sum_{k \in J} p_{k}\right]^{q}\right\rangle=\sum_{J=1}^{[A / L]}\left\langle\left[\sum_{k \in J} k n_{k} / A\right]^{q}\right\rangle,
$$

of order 2 analytically. Using the relations developed in Refs. [2, 3], 


$$
\begin{aligned}
P_{2}(L, x) & =\sum_{J=1}^{[A / L]}\left\langle\left[\sum_{k \in J} k n_{k} / A\right]^{2}\right\rangle=\sum_{J=1}^{[A / L]} \sum_{j \in J} \sum_{k \in J} j k<n_{j} n_{k}>/ A^{2} \\
& =\sum_{k=1}^{A} \frac{k^{2}<n_{k}>}{A^{2}}+\sum_{k=1}^{[A / 2]} \frac{k^{2}<n_{k}\left(n_{k}-1\right)>}{A^{2}}+\sum_{J=1}^{[A / 2 L]} \sum_{j \in J} \sum_{k \neq j \in J} \frac{j k<n_{j} n_{k}>}{A^{2}} \\
& =\frac{(A+x)}{A(x+1)}+\sum_{J=1}^{[A / 2 L]} \sum_{j \in J} \sum_{k \in J} \frac{x^{2}}{A^{2}} \frac{A !}{(A-j-k) !} \frac{\Gamma(A-j-k+x)}{\Gamma(A+x)} .
\end{aligned}
$$

Thus we have

$$
\begin{aligned}
& P_{2}(L, 1)=\frac{1}{2}\left[\frac{(A+1)}{A}+\frac{L}{A}\right] \\
& P_{2}(L, 2)=\frac{1}{3}\left[\frac{(A+2)}{A}+3 \frac{L}{(A+1)}\right] \\
& P_{2}(L, 3)=\frac{1}{4}\left[\frac{(A+3)}{A}+\frac{3\left(2 A^{2}+3 A-1\right)}{A(A+1)(A+2)} L-\frac{3}{A(A+1)(A+2)} L^{3}\right] .
\end{aligned}
$$

Eq.(35) is valid for bin lengths $L$ which divide $A$ into an "integer" number of parts (except $L=A$, thus $L \leq A / 2)$. These results show a linear dependence of the second order $(q=2)$ power moments in $L$ rather than a pure power law or exponential behavior (see Fig.5). When the $L$ independent part of $P_{2}(L, m)$ is subtracted out, the resulting quantity has a power law behavior in $L$ with an exponent of 1 . When the constant part is accepted, these $P_{2}(L, m)$, $m=1,2,3$, can be approximated as an exponential. In Ref. [8], we have shown that the power moments follow approximately the power law behavior at large $x$ and exponential behavior at a small value of $x$. The saturation at large bin size $L$ for large $x$ comes from the high power dependences in $L$, such as the $L^{3}$ term in $P_{2}(L, 3)$.

On the other hand, for a bin size of 1 ,

$$
\begin{aligned}
P_{2}(1, x) & =\sum_{k=1}^{A}\left\langle\left[\frac{k n_{k}}{A}\right]^{2}\right\rangle=\sum_{k=1}^{A}\left[\frac{k}{A}\right]^{2}<n_{k}>+\sum_{k=1}^{A}\left[\frac{k}{A}\right]^{2}<n_{k}\left(n_{k}-1\right)> \\
& =\frac{(A+x)}{A(1+x)}+\sum_{k=1}^{[A / 2]}\left[\frac{x}{A}\right]^{2}\left[\frac{A !}{(A-2 k) !} \frac{\Gamma(A+x-2 k)}{\Gamma(A+x)}\right] .
\end{aligned}
$$

Since $<m_{k}>=k<n_{k}>$ is small for large $k$ at a large value of $x$, then using Eq.(22), the $P_{2}(1, x)$ can be approximated as

$$
P_{2}(1, x) \approx \frac{(A+x)}{A(1+x)}+\left[\frac{x}{A+x-1}\right]^{2}\left[\frac{1-\left(\frac{A}{A+x-1}\right)^{A}}{1-\left(\frac{A}{A+x-1}\right)^{2}}\right]
$$


For large $A>>x$ with Stirling approximation Eq.(22),

$$
P_{2}(1, x) \approx \frac{(A+x)}{A(1+x)}+\frac{x}{2 A}+\frac{x^{2} \Gamma(x)}{A^{x+1}} .
$$

For large $A$ with $x<<A$, the leading order $L / A$ dependence in $P_{2}(L, x)$ is approximately (c.f. Eq.(35))

$$
P_{2}(L, x)=\frac{1}{x+1}+\frac{x}{A(x+1)}+C_{2} \frac{L}{A}
$$

where

$$
C_{2} \approx \frac{x}{2}+\frac{x^{2} \Gamma(x)}{A^{x}}
$$

Writing $P_{q}(L, x) \propto e^{q L / \zeta_{q}}$ as in Ref. [8], the correlation length $\zeta_{q}$ for $q=2$ is

$$
\zeta_{2}=\frac{2(A+x)}{(x+1) C_{2}}
$$

This formula gives value for $\zeta_{2}$ which approximately agree with the results of Table 2 in Ref. 8] for small values of $x$.

\section{FOURIER TRANSFORMS OF MASS DISTRIBUTION AND THE $1 / f^{\beta}$ POWER LAW}

In this section we propose that Fourier spectra of fragmentation distributions be studied and we illustrate the procedure with an example. The importance of Fourier or power spectrum is the possible appearance of the ubiquitous $1 / f$ noise which appears in many areas of physics. Various types of noise can be characterized by writing the power spectrum associated with the noise as $1 / f^{\beta}$, with $\beta=0$ for white, $\beta=1$ for pink, $\beta=2$ for brown, and $\beta>2$ for black noise. One mechanism for generating $1 / f$ noise is through intermittency generated by the logistic map near its tangent bifurcation [9]. The possible role of the logistic map in nuclear fragmentation was noted in Section III. The Malthus model considered in this section will generate a $1 / f^{2}$ power spectrum. This example is used for the purpose 
of illustrating a powerful tool used in studying properties of discrete dynamical systems through Fourier analysis.

The dynamical system considered here will be the set of mass points $m_{k}$ for $k=1,2, \ldots$, $A$ or its associated probability $p_{k}=m_{k} / A$ which are generated by an iterative map given by Eq.(8). The Fourier transform of $m_{k}$ gives the frequency spectrum of the mass distribution;

$$
\begin{aligned}
\mathcal{M}_{f} & =\sum_{k=1}^{A} p_{k} \exp \left[-i \frac{2 \pi f}{A} k\right] \\
& =\frac{1}{A} \sum_{k=1}^{A} m_{k} \exp \left[-i \frac{2 \pi f}{A} k\right]
\end{aligned}
$$

For the mass distribution of Eq.(8), we have

$$
\begin{aligned}
\mathcal{M}_{f} & =\frac{m_{1} / r}{A} \sum_{k=1}^{A}\left[r e^{-i 2 \pi f / A}\right]^{k} \\
& =\frac{m_{1}}{A}\left(1-e^{-i 2 \pi f} r^{A}\right) \frac{e^{-i 2 \pi f / A}}{1-r e^{-i 2 \pi f / A}} .
\end{aligned}
$$

Notice here $\left(e^{-i 2 \pi f / A}\right)^{A}=e^{-i 2 \pi f}=1$ for any integer $f$. An important quantity associated with $\mathcal{M}_{f}$ is the intensity or power spectrum;

$$
\begin{aligned}
I_{f}=\left|\mathcal{M}_{f}\right|^{2} & =\frac{m_{1}^{2}}{A^{2}}\left[\frac{1+r^{2 A}-2 r^{A} \cos (2 \pi f)}{1+r^{2}-2 r \cos (2 \pi f / A)}\right] \\
& =\frac{m_{1}^{2}}{A^{2}}\left(\frac{1-r^{A}}{1-r}\right)^{2}\left[\frac{1+\frac{2 r^{A}}{\left(1-r^{A}\right)^{2}}(1-\cos (2 \pi f))}{1+\frac{2 r}{(1-r)^{2}}\left(1-\cos \left(\frac{2 \pi f}{A}\right)\right)}\right] .
\end{aligned}
$$

The intensity $I_{f}$ peaks at half integer $f$ and has a valley at each integer $f$. For a Fourier transform in general, $\mathcal{M}_{f}$ and $I_{f}$ are periodic in $f$ with the period of $A$. Furthermore, $I_{f}$ has a reflection symmetry with respect to $A / 2$, i.e., $I_{A-f}=I_{f}$. Here we consider only integer $f$, i.e., the valleys of Eq.(42) for $0 \leq f \leq A / 2$. At integer $f$, Eq.(42) reduces to

$$
I_{f}=\left|\mathcal{M}_{f}\right|^{2}=\frac{m_{1}^{2}}{A^{2}}\left(\frac{1-r^{A}}{1-r}\right)^{2}\left[1+\frac{2 r}{(1-r)^{2}}\left(1-\cos \left(\frac{2 \pi f}{A}\right)\right)\right]^{-1} \text {. }
$$

For an infinitely large $A$, except for $r=1, I_{f} \propto f^{0}$ for finite $f$ which is a power law in $f$ with the exponent of zero. This result represent the self-similar property of the Malthus map discussed in the previous section. When $r=1$, which is the same as the $x$ model at $x=1$, 
Eq.(43) shows that $I_{f}=0$ except for values of $f$ which are integer multiple of $A$. For $f=A$, $I_{A}=m_{1}^{2}=1$ since $m_{k}=1$. For $r$ very small or large compared to one which corresponds to a large or small value of $x$ in the $x$ model, the spectrum $I_{f}$ becomes independent of the frequency $f$ and thus has a white power spectrum [9]; if $\epsilon=r-1>2 \pi f / A$, then $I_{f}$ of Eq.(43) exhibits a white spectrum. On the other hand, for $r$ near unity, the power spectrum for small $f$ compared to $A$ can be approximated as $I_{f} \propto f^{-2}$. The conditions for a $1 / f^{2}$ power spectrum are, for $\epsilon=r-1<<1$,

$$
\epsilon^{2}=(r-1)^{2}<<\left[\frac{2 \pi f}{A}\right]^{2}<<1 .
$$

In Fig. 1 of Section II, the choice of $r \approx 1.02$ fit the proton-nucleus data for $A \approx 200$. The $\epsilon A \sim 4$ and the data exhibits $1 / f^{2}$ behavior for $1<f<30$ (see Fig.9(a) in the following Sec-

tion VI). The steepest slope occurs at $f=A / 4$ and $3 A / 4$ with $\pm\left(\frac{m_{1}}{A}\right)^{2}\left(\frac{1-r^{A}}{1-r}\right)^{2}\left(\frac{(1-r)^{2}}{1+r^{2}}\right)^{2} \frac{4 \pi r}{A}$.

As a final illustration, we consider the power spectrum associated with the result of Eq.(11) at the point $x=2$. Substituting $m_{k}=k<n_{k}>$ into Eq.(40) we obtain

$$
I_{f}=\frac{1}{(A+1)^{2} \sin ^{2}(\pi f / A)}
$$

for $f=1,2, \ldots A-1$. With low frequencies such that $\pi f / A<<1, I_{f} \sim 1 / \pi^{2} f^{2}$ which is a $1 / f^{\beta}$ behavior with $\beta=2$. A more detailed analysis of $1 / f^{\beta}$ power spectrum in mass distribution will be given in the next section using the Au emulsion data of Ref. [10].

\section{AU EMULSION DATA}

In this section we will discuss the Au emulsion data of Ref. [10]. The Au emulsion data is an event by event analysis of the charge distribution $n_{z}$, with $n_{z}$ the number of charged $z$ fragments produced in the collision in each event. By contrast, the data considered in Fig.1 is an ensemble average distribution. Each event conserves the total charge $Z=79$ event by event in the $\mathrm{Au}$ data.

The ensemble averaged element distribution $\left\langle n_{z}>\right.$ is shown in Fig.6(a) and the mean charge distribution $p_{z}=<m_{z}>/ Z$ is shown in Fig.6(b). These show the strong even-odd 
oscillation in the data (filled circles connected with a thin solid line). Here we mention that the charge distribution $\left\langle m_{z}>\right.$ may be better than the element distribution $\left\langle n_{z}\right\rangle$ in distinguishing between different model. Unlike the results of Fig.1, the Au data cannot be fit with a single value of $x$ or $r$. The reason for this is the initial fast fall off with $z$ in $n_{z}$ or $m_{z}$. Fig.6(b) show that two mixed $x$ 's in the $x$ model (solid line) or three mixed $r$ 's in the Malthus model (dash-dotted line) give a good fit to the data; both exhibit the fast drop of $\left\langle m_{z}\right\rangle$ at small $z$ followed by a flat middle range and then a rise at the large $z$. Also shown in Fig.7(a) is the multiplicity distribution $P(M)$ where the multiplicity $M=\sum_{z} n_{z}$, event by event. This figure shows that the emulsion data does not corresponds to a single $x$ nor a single $r$.

In Refs. [1],3,8], the importance of the cumulative mass distribution

$$
M(z)=M_{z}=\sum_{j=1}^{z} m_{j}
$$

was also stressed. The $M_{z}$ has a staircase behavior and the sequence $M_{1}, M_{2}, \ldots, M_{z}, \ldots$, $M_{Z}$ can be considered as an ordered sequence similar to the sequence of "energy" levels [8]. If we use the mean distribution $\left\langle m_{z}>\right.$ in Eq.(46), then, at $x=1$ or $r=1$, levels are uniformly spaced with a spacing $s=1$. The distribution of the experimental "spacings" is then determined by the experimental values of $m_{k}=k n_{k}$ event by event which may then be compared with a Poisson or a Wigner distributions. Fig.7(b) shows the $m_{z}$ spacing distribution in the $\mathrm{Au}$ emulsion data (solid circle connected by a thin line). Here $m_{z}$ is the spacing in each event and the probability is the ensemble averaged one. The data looks more like a Wigner distribution $\left(m_{z} e^{-m_{z} / 5} / 25\right)$ than a Poisson spacing $\left(e^{-m_{z} / 10} / 10\right)$. In level spacings, the Wigner distribution is discussed in terms of a chaotic structure in the spacing distribution. It should also be noted that the staircase feature [8] of the cumulative mass distribution of the data (thin solid line in Fig.8(a)) can be described by two $x$ 's (thick solid line) or by three r's (dash-dotted line). These three curves are quite similar as can be seen in the figure.

In Fig.8(b) we show a cobweb type plot $\left(m_{z} v s m_{z+1}\right)$ by solid circles. Also shown in 
the figure are the pairs of $\left(m_{z}, m_{z+2}\right)$. The origin of the scatter of the points is the evenodd oscillating behavior which also has a varying amplitude in the charge distribution of Fig.6(b). If the data follows the $x$ model or the Malthus model, then the points would lie on a single line. The scattered points indicate that even the mean distribution of the data exhibits some random structure.

Finally, we show the Fourier spectrum of the data in Fig.9. Fig.9(a) shows the power spectrum of the data compared with the power spectrum of the $x$ model and the Malthus model. In the low frequency region, the $x$ model with $x=0.3$ (dashed line) exhibits a $f^{-\beta}$ behavior with $\beta \approx 0.56$, the Malthus model with a single $r$ (dotted and dash-dot-dot-dotted lines) has $\beta \approx 1.9$, and the Malthus model with three mixed $r$ 's (dash-dotted line) follows a power law with $\beta \approx 0.68$. The data (solid circle connected by a thin line) shows a more complicated structure than the $x$ model or the Malthus model. Also shown by open circles connected with a thin dashed line is the Fourier spectrum of the data in which the high peak at $z=1$ and 2 in the $m_{z}$ distribution is reduced by putting these values at the value of $m_{3}$. This spectrum then follows the single Malthus model at the low frequency part. In Fig.9(b), we show the $f$ dependence of the fluctuations of the data from the mean behavior given by the $x$-model. The thin dashed line is for $x=3$, the thick solid line is computed with $2 x$ 's $(66 \% x=0.5$ and $34 \% x=50)$, and the thin solid line is the Malthus model with three $r^{\prime}$ s. This figure does not exhibits a simple $1 / f^{\beta}$ behavior.

\section{CONCLUSION}

In conclusion, this paper illustrates a simple approach to the distribution of mass produced in a heavy ion collision. This distribution can be considered in terms of iterative one dimensional maps similar to those used in population dynamics. In this picture, the orbit of a monomer or nucleon in its approach to an attractor of the dynamics generates the mass distribution. Simple Malthusian dynamics seem to account for some features of the data. A non-linear model is also discussed to see if the data has any evidence for non-linearities 
and limiting behavior in otherwise geometric growth. When the Verhulst model is used to generate the mass distribution $m_{k}$, the resulting distribution of cluster sizes $n_{k}=m_{k} / k$ is shown to have an interesting bifurcation point whose signatures are discussed. New ways of representing data which go beyond any specific model are also suggested. These ways include plotting pairs $\left(m_{k}, m_{k+1}\right)$ which appear in cobwebbing, plotting mass yields, cumulative mass yields, and inverse mass yields versus cluster size $k$ besides the traditional yields, $n_{k}=m_{k} / k$ versus $k$. More complex maps can also be investigated than those discussed here as well as fluctuations such as in Ref. [8].

Using the simple geometric model developed in this paper, questions related to selfsimilarity and scaling behavior are discussed. Simple scaling relations for the power moments of the mass distribution are developed based on self-similar geometric properties of the mass yields. The correlation lengths, exponents and dimensions associated with these scaling relations are discussed. The power spectrum associated with Fourier transforms of the mass spectrum is also developed. Under certain conditions a $1 / f^{\beta}$ spectrum is found.

One of us (A.M.) would like to thank J.D. Murray of the University of Washington for his many lectures on methods in mathematical biology. He would also like to thank E. Henley and L. Wilets for the hospitality offered him at the Institute of Theoretical Physics where this research was started. This research was supported in part by the N.S.F. grant number NSF89-03457 and by the DOE. 


\section{REFERENCES}

[1] A.Z. Mekjian, Phys. Rev. C41, 2103 (1990); Phys. Rev. Letts. 64, 2125 (1990).

[2] S.J. Lee and A.Z. Mekjian, Phys. Rev. C45, 1284 (1992).

[3] A.Z. Mekjian and S.J. Lee, Phys. Rev. A44, 6294 (1991).

[4] J.D. Murray, Mathematical Biology, Biomathematics V.19, (Springer-Verlag, 1989).

[5] F.C. Hoppensteadt, Mathematical Methods of Population Biology, (Cambridge University Press, 1982).

[6] R. Devaney, Chaos and Fractal, Proceedings of Symposia in Applied Mathematics, V.39, (R. Devaney and L. Keen, eds., American Mathematical Society, 1989).

[7] J.E. Cohen, Casual Groups of Monkeys and Man (Harvard University, Cambridge, MA, 1971);

Theor. Popul. Biol. 3, 119 (1972).

[8] S.J. Lee and A.Z. Mekjian, to be appear in Phys. Rev. C, "Self-Similarity and Scaling Behavior ..."

[9] M. Schroeder, Fractals, Chaos, Power Laws; Minutes from an Infinite Paradise, (W.H. Freeman and Company, New York, 1991).

[10] C.J. Waddington and P.S. Freier, Phys. Rev. C31, 888 (1985).

[11] S.J. Lee and A.Z. Mekjian, Phys. Rev. C45, 365 (1992).

[12] D.H.E. Gross, L. Satpathy, M. Ta-chung, and M. Satpathy, Z. Phys. A309, 41 (1982).

[13] A.Z. Mekjian, Phys. Rev. A44, 8361 (1991).

[14] W.J. Ewens, Theoretical Population Biol., 3, 87 (1972);

Mathematical Population Genetics, (Springer-Verlag, Berlin, 1979). 


\section{FIGURES}

FIG. 1. Fragment mass distributions in the radiochemical measurements from the decay of a target residue produced in a collision of high energy proton on a nucleus. The filled circles are the data summarized in Ref. [12] and the histogram are the thermodynamic statistical fits of Ref. [12]. The solid curve is obtained using the $x$-model of Eq.(1) with $x=0.3$ and the dashed line is the orbit under the Malthus linear iterative map of Eq.(8). The coefficients $r$ for the Malthus map are $r=1.065$ in (a), 1.027 in (b), 1.024 in (c), and 1.041 in (d). These values are the geometric mean of the corresponding $r_{A}(k, x)$ with $x=0.3$ in all four cases. The dash-dotted line in (c) is a linear map with the recursive coefficient $r_{k}=1+(k-50) / 3000$. The dash-dotted line in $(\mathrm{d})$ is an orbit in the non-linear Verhulst map Eq.(10) with $\zeta=0.062, m_{s}=170$, and $m_{1}=1$.

FIG. 2. The power moments $P_{q}(L, r)$ of the mass distribution in a Malthusian model. The mapping coefficients $r$ for each $x$ are $r=A /(A+x-1)$ with $A=79$. The solid, dashed, dotted, and dash-dotted lines are for $q=2,3,4$, and 5 respectively.

FIG. 3. The power moments $\bar{P}_{q}(L, x)$ of the mean distribution in the $x$ model $v s$ the power moments $P_{q}(L, r)$ of the Malthus model of Fig.2 with the corresponding $x$ values. The lines are same as in Fig.2.

FIG. 4. Power moments $\bar{P}_{q}(L, x)$ of the mean distribution of mass in the $x$ model for $x=0.5$ and $A=79$ (a) and their corresponding slopes, $\alpha_{q}(L)=(q-1) D_{q}(L)$ using Eq.(20) (b). The solid, dashed, dotted, and dash-dotted thick lines are for $q=2,3,4$, and 5 respectively. The thin solid lines in (a), which are close to the thick lines, are fits using a form $\bar{P}_{q}(L)=(L / A)^{\left(a_{q}+b_{q} / L\right)(q-1)}$ and

the thin dashed lines are the simple power law fit of $\bar{P}_{q}(L)=(L / A)^{D_{q}(q-1)}$. The thin dash-dotted line, which is close to thin dashed line, is based on Stirling's approximation for $q=2$, Eq.(23). Here, $a_{q}=D_{q}-b_{q}$ with $b_{q}=0.04$ and $D_{1}=0.942, D_{2}=0.849, D_{3}=0.756, D_{4}=0.695$, and $D_{5}=0.656$. 
FIG. 5. Power moments $P_{q}(L, x)$ which are the ensemble average of the power of the mass distribution in $x$ model for $A=79$. The solid and dashed lines are for $q=2$ and 3 respectively. The dotted lines for $x=1.0$ and 3.0 are the exact results of Eq.(35) and the dotted lines for $x=0.5$ and 5.0 are the approximation of Eq.(37).

FIG. 6. The cluster distribution (a) and the charge distribution (b) of Au emulsion data $(Z=79)$ of Ref. [10]. The solid circle connected by a thin line is the data. The dashed line is the $x$ model with $x=0.3$ and the solid line is the $x$ model with a mixture of 0.66 of $x=0.5$ and 0.34 of $x=50$. The dotted line is the Malthus model with $r=Z /(Z+x-1)$ with $x=0.3$ and the dash-dot-dot-dotted line is for $r$ given by geometrical mean of $x$ model; the corresponding $r$ values are 1.0089 and 1.0546 respectively. The dash-dotted line is obtained from a mixture of three $r$ 's: $1.1210(29 \%), 0.9938(40 \%)$, and $0.3492(31 \%)$ respectively.

FIG. 7. The multiplicity (a) and the "level spacing" (b) distributions in the ensemble of 415 $\mathrm{Au}$ data events (the solid circle connected by a thin line). In (a), the dashed line is the $x$ model with $x=0.5$, the solid line is the mixture of 0.66 of $x=0.5$ and 0.34 of $x=50$, and the thin solid line is the mixture of 0.72 of $x=0.5,0.15$ of $x=5$, and 0.13 of $x=79$. The dashed and solid lines in (b) are Poissonian $\left(e^{-m_{k} / 10} / 10\right)$ and Wigner $\left(m_{k} e^{-m_{k} / 5} / 25\right)$ spacing distribution respectively.

FIG. 8. Staircase (a) and cobweb (b) plots. In (a), the curves are the same as in Fig.6 except the thin solid line is for the data which is very close to the dash-dotted line and to the thick solid line. In (b), the solid circles are the pairs of $\left(m_{z}, m_{z+1}\right)$ and the closes are the pairs of $\left(m_{z}, m_{z+2}\right)$. 
FIG. 9. The Fourier transform of the charge distribution $p_{z}(\mathrm{a})$ and the noise spectrum (b) for the Au data. The curves in (a) are the same as in Fig.6. The open circles connected by a thin dashed line, are the Au emulsion data except that the charge distribution at $z=1$ and 2 are each taken the same as the $z=3$ value. This is done to reduce the effect from the large probability of charge number 1 and 2. In (b), the thin dashed and the thick solid lines are the noise spectrum of the Au data with respect to the $x$ model with a single $x(x=0.3)$ and for two mixed $x$ 's $(0.66$ of $x=0.5$ and 0.34 of $x=50$ ) respectively. The thin solid line is the noise spectrum with respect to the Malthus model with three $r$ 's as in Fig.6. Also shown by the solid circles connected with a thin line is the power spectrum of the data which is shown in (a). 